\title{
Filterless, Digital Class-BD Audio Amplifiers with Constant Common Mode Output Voltage
}

\author{
Stanisław Kuta ${ }^{1}$, Witold Machowski ${ }^{2, *}{ }^{\mathbb{D}}$, Wojciech Kołodziejski ${ }^{1}\left(\mathbb{D}\right.$ and Lukasz Chlastawa $^{1}$ \\ 1 Polytechnic Faculty, University of Applied Sciences in Tarnów, Mickiewicza 8, 33-100 Tarnów, Poland; \\ st_kuta@pwsztar.edu.pl (S.K.); w_kolodziejski@pwsztar.edu.pl (W.K.); 1_chlastawa@pwsztar.edu.pl (Ł.C.) \\ 2 Faculty of Computer Science, Electronics and Telecommunications, Institute of Electronics, AGH University \\ of Science and Technology, Mickiewicza 30, 30-059 Kraków, Poland \\ * Correspondence: witold.machowski@agh.edu.pl
}

check for

updates

Citation: Kuta, S.; Machowski, W.; Kołodziejski, W.; Chlastawa, Ł.

Filterless, Digital Class-BD Audio Amplifiers with Constant Common Mode Output Voltage. Energies 2021, 14, 7014. https://doi.org/10.3390/ en14217014

Academic Editors: Lubomir Bena,

Damian Mazur, Bogdan Kwiatkowski and Teuvo Suntio

Received: 18 September 2021

Accepted: 19 October 2021

Published: 26 October 2021

Publisher's Note: MDPI stays neutral with regard to jurisdictional claims in published maps and institutional affiliations.

Copyright: (c) 2021 by the authors. Licensee MDPI, Basel, Switzerland. This article is an open access article distributed under the terms and conditions of the Creative Commons Attribution (CC BY) license (https:// creativecommons.org/licenses/by/ $4.0 /)$.

\begin{abstract}
This paper presents new and original architectures and implementations of two filterless, open-loop digital Class-BD audio amplifiers with constant common mode (CM) output voltage. The first, low-power amplifier consists of four ultrahigh-current EL7158 pin drivers, the OUT pins of which form the $\mathrm{H}$ bridge. The drivers are controlled from the extended LBDD PWM modulator and have appropriately set voltage levels on the VL and VH pins. The second proposed amplifier consists of two cooperating H-bridge power stages (HBS) implemented on complementary MOSFET pairs and powered by two different voltages. Both HBSs are driven by four EL7158 pin drivers having appropriately adjusted voltage levels on the VL and VH pins and controlled by an extended LBDD PWM modulator. The hybrid 9 bit DPWM modulator uses a linearized Class-BD doublesided modulation scheme with pre-compensation, enabling the most faithful emulation of natural NBDD PWM and, thus, providing attractive spectral characteristics at the DM output. Basic SPICE simulations and experimental results of the proposed digital Class-BD amplifiers were compared with the conventional digital Class-BD amplifiers. The elimination of CM signals significantly contributes to the reduction in electromagnetic interference (EMI), particularly those produced on the cables connecting the loudspeaker to the HBS terminal. Proposed Class-BD amplifiers with original and new topologies, due to their simple design and easy control (no galvanic isolation of control signals and no floating bias power supplies), which generate no CM voltage and feature excellent DM performance, similar to conventional Class-BD amplifiers, are very attractive solutions for filterless Class-BD amplifiers.
\end{abstract}

Keywords: digital Class-D audio amplifier; filterless Class-D amplifier; linearized pulse width modulation (LPWM); electromagnetic interference (EMI)

\section{Introduction}

Much work has been done on various kinds of improved PWM methods, as well as Class-D power amplifier topologies to achieve high energy efficiency, high fidelity, small size, and reduced power dissipation. Improving these performances is especially important in battery powered portable devices. However, the full implementation of Class$\mathrm{D}$ technology in portable devices required solving many problems in order to improve electroacoustic, energy, and electromagnetic compatibility (EMI) performance and filter the output signal [1-5].

As with analog Class-D amplifiers, the architectures of digital Class-D amplifiers can generally be divided into two categories: open-loop Class-D amplifiers with digital pulse width modulation (DPWM) and closed-loop ones with sigma-delta modulation (SDM) and a bang-bang controller.

Depending on the sampling method, DPWM schemes can be divided into two main classes: uniform PWM (UPWM) and linearized PWM (LPWM). In addition, each DPWM 
sampling method also distinguishes one-edge or two-edge DPWM, as well as two-level (Class-AD) or three-level (Class-BD) switching [6].

UPWM modulation would be the easiest to implement in an open-loop Class-D digital amplifier; however, its primary problem is inherent nonlinearity. The use of pre-correction algorithms and increasing the sampling frequency of the input signal lead to a reduction in this nonlinearity to an acceptable level, but with higher power dissipation [6,7]. Adoption of a ternary UPWM modulation scheme enabled the implementation of the filterless highperformance digital Class-D amplifier [8].

Linearized PWM (LPWM) with pre-compensation algorithms is based on the most faithful emulation of the analog NPWM with natural sampling.

Similar to analog NPWM modulation schemes, among four basic LPWM methods, LBDD PWM (linearized Class-BD double sided PWM), equivalent to the linearized phaseshifted carrier PWM (LPSC PWM), exhibits very attractive spectral characteristic at the differential mode (DM) output, containing much fewer unwanted IMD products than other LPWM methods. However, the LBDD PWM signal on the CM output reveals some drawbacks, as its wide frequency spectrum contains many undesirable odd harmonics of the switching frequency and their even IMD products. For this reason, the LC filter for Class-BD amplifier with LBDD PWM modulation must be designed separately for DM and CM components [7].

The Class-D amplifier operating in switching mode at high frequency contributes significant electromagnetic interference (EMI). One of the major sources of EMI is represented by the sharp spikes of the shoot-through currents, generated during the switching operation of the power MOSFETs. These short current pulses occur at the rising and falling edges of the DPWM signal, as well as on the power supply bus. Another source of EMI is represented by CM signals, in particular those produced on the cables connecting the loudspeaker to the BTL terminals.

Shoot-through currents can be reduced by adding dead times in front of the edges of the control signals switching on the power transistors, thus also reducing EMI. The addition of dead times and the associated elimination of or reduction in shoot-through currents increases the efficiency of the amplifier, as well as reduces EMI, but simultaneously introduces additional nonlinear distortion of the audio output signal; therefore, a compromise is necessary to obtain acceptable distortion and efficiency of the amplifier.

Various techniques to reduce the EMI of Class-D amplifiers have been reported [8-13]. An innovative multilevel PWM was presented in [14], which was implemented by converting the two-level PWM signal directly into a multilevel signal using the original time-division adder. An integrated Class-D amplifier with an ultralow level of EMI, using a feed-forward ADC and feedback filters, was presented in [15]. Another method of reducing EMI in Class$\mathrm{D}$ amplifiers is the use of pseudorandomized carrier frequency modulation (PRCFM) [16].

The organization of this paper is as follows: Section 2 presents implementation of the digital Class-BD amplifier using a 9 bit hybrid LBDD modulator. Section 3 presents the original architectures and implementations of the digital version of the Class-BD audio amplifiers with constant voltage at the $\mathrm{CM}$ output, which are controlled by an extended hybrid modulator, based on the LBDD PWM modulation scheme. Section 4 presents basic SPICE simulations and experimental results of the proposed digital Class-BD amplifiers implemented on ultrahigh-current EL7158 pin drivers and complementary MOSFET pairs. Discussion and conclusions are given in Sections 5 and 6, respectively.

\section{The Digital Class-BD Amplifier Using 9 Bit Hybrid LBDD Modulator}

Figure 1 shows the general block diagram of an open-loop digital Class-D amplifier with LPWM digital modulation and pre-compensation, making it possible to emulate all four basic NPWM modulation methods [7].

The input audio signal is converted into an $N_{q}$-bit PCM data stream, which the digital modulator converts directly into a train of 1 bit physical DPWM pulses. In the interpolation process, additional signal samples uniformly distributed over the switching period $T_{\mathcal{c}}$ are 
added into the PCM data stream at the ADC output. The modulating signal in this interval is approximated by rectilinear segments passing through the interpolated PCM samples. The digital values of the pulse-edge locations, the $t_{p}(n)$ rising edge and the $t_{k}(n)$ falling edge of the LPWM pulse within $n$-th period $T_{c}$ of the switching frequency, calculated with a high $N_{q}=32$ bit resolution, are then requantized to a lower $N_{r q}$-bit resolution $\left(N_{r q}<N_{q}\right)$, to moderate an excessive requirement of high clock frequency [7]. At the calculated pulseedge locations requantized to a 9 bit resolution, it is possible to implement a digital-to-time converter (DTC) on the basis of the hybrid method, to convert the calculated data into the physical train of the LBDD PWM pulses.

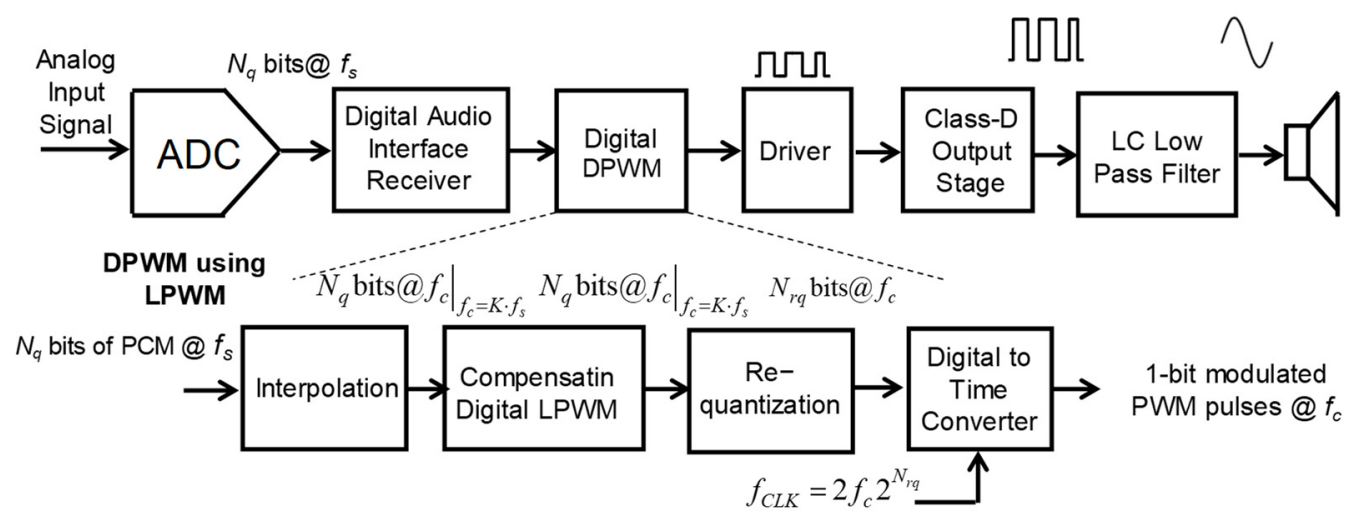

Figure 1. Block diagram of an open-loop digital Class-D amplifier.

The requantized data are split into two parts consisting of 6 MSB (8:3) and 3 LSB (2:0) data bits; then, each $n$-th period of the switching frequency is sequentially rewritten into LADD registers. The $6 \mathrm{MSB}$ data are converted on the basis of the counter method using an STM32 microcontroller with its peripherals, while the remaining 3 LSB data are converted using a method based on the integrated 3 bit FAST TTL PTLDL [17].

The timing of the signal processing blocks in Figure 1 is based on the oscillator frequency of the three microcontroller AHB buses [18].

The external converter based on the 3 LSB (2:0) PTD is controlled by the frequency.

$$
f_{P C L K 2}=f_{C L K h}=\frac{f_{\text {osC }}}{P_{1}}=\frac{168 \mathrm{MHz}}{4}=42 \mathrm{MHz} .
$$

The conversion frequency $f_{C O N V}$ of the ADC [18] and the switching frequency are the same.

$$
f_{\mathrm{CONV}}=f_{\mathrm{c}}=\frac{f_{\mathrm{CLKh}}}{2^{\left(N_{r q}-m\right)+1}}=\frac{42 \mathrm{MHz}}{2^{7}}=328.125 \mathrm{kHz} .
$$

The delay time $t_{d 1}$ of one 3 bit PTDL segment, consisting of eight identical delay segments, results from the division of the clock period $T_{C L K h}$ into eight parts.

$$
t_{d 1}=\frac{T_{C L K h}}{2^{3}}=\frac{1}{f_{C L K h}} \cong 2.97 \mathrm{~ns} .
$$

The conversion of the calculated and requantized 3 LSB LADD PWM audio data into a sequence of 1 bit LADD PWM pulses is shown in Figure 2. Using 6 MSB (8:3) bits of the requantized values of the rising edge $t_{p}(n)$ and the falling edge $t_{k}(n)$ locations, which are stored in LADDL registers, the STM32 microcontroller and its advanced-control timers generate in each $T_{c}$ period two synchronous sequences of the linearized Class-AD single sided PWM (LADS PWM) signal. The falling edges of both generated LADS PWM signals are differentiated and then passed through a 3 bit PTDL. As a result, at the output of PTDL we get the spike pulses, which are delayed by the time programmed twice in each switching period $n T_{c}$ for the 3 LSB (2:0) bits of the requantized values of the rising edge $t_{p}$ $(n)$ and the falling edge $t_{k}(n)$ locations. 
The delated spike pulses corresponding to the rising edge $t_{p}(n)$ locations, which occur at the PTDL output in the first half of the switching cycle $T_{c}$, set the RS output flip-flop, while the pulses that occur in the second half of the switching cycle $T_{c}$, which correspond to the falling edge $t_{k}(n)$ locations, reset this flip-flop. As a result, at the output of the flip-flop, the final 9 bit LADD PWM signal is generated.
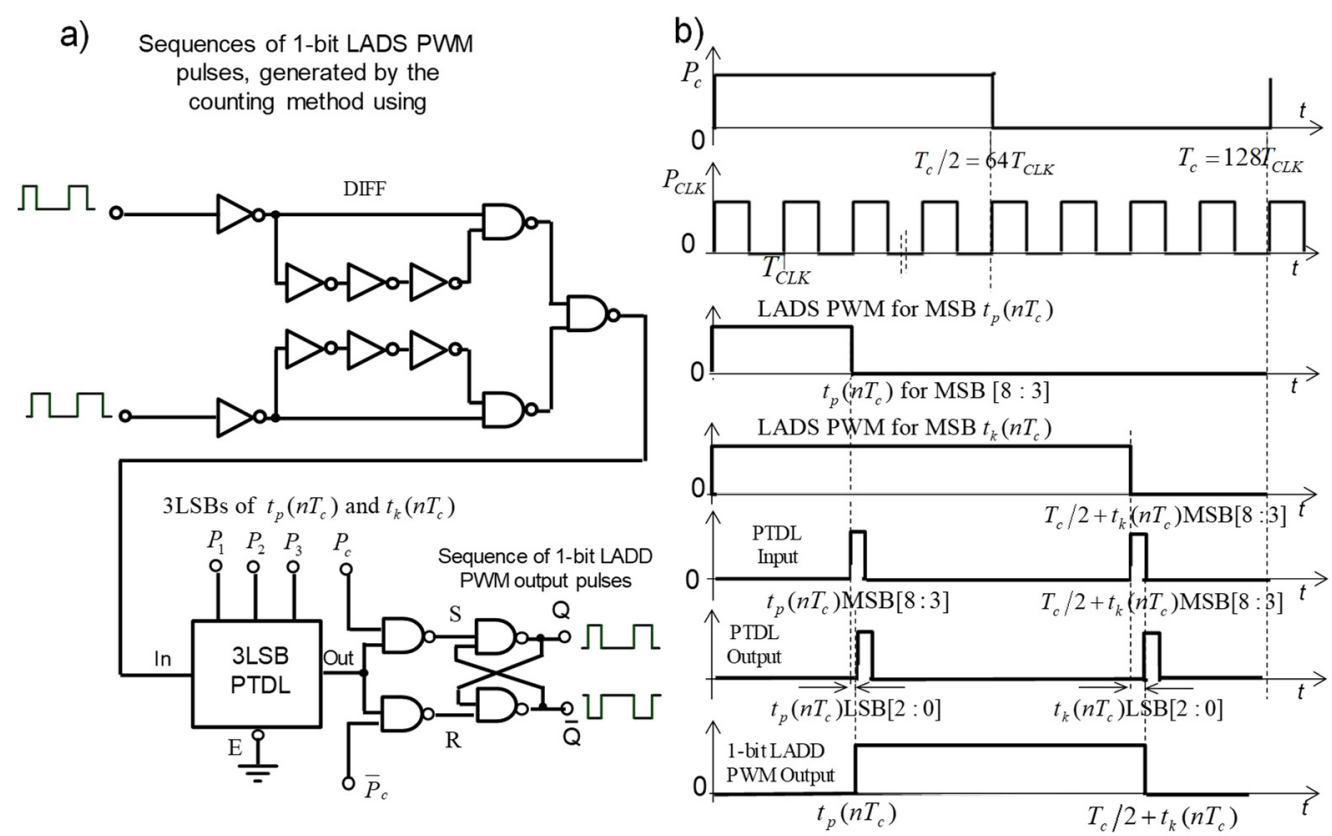

Figure 2. Conversion of the calculated and requantized 3 LSB LADD PWM audio data into a sequence of 1 bit LADD PWM pulses: (a) logical scheme of the LADD subsystem; (b) the control and output signals.

Figure 3 shows the HBS implemented on the IRF9389PbF power MOSFET pairs, which are driven by the EL7158 high-performance inverting drivers. The left branch of the $\mathrm{H}$ bridge is controlled by the $L A D D_{L} P W M$ signal generated for the direct audio signal, while the right branch of the H-bridge is controlled by the $L A D D_{R} P W M$ signal generated in the same way for the inverted audio signal. Shoot-through currents can be reduced by adding dead times in front of the edges of the control signals switching on the power transistors, thus also reducing EMI [7]. These times are independently set on the rising edges of the signals driving of NMOSFETs (the $R_{G N}$ resistors) and on the falling edges of the signals driving PMOSFETs (the $R_{G P}$ resistors). Because the gate drivers and the HBS implemented on complementary MOSFETs are powered by the same voltage, a level shifter of the gate driver output signal is needed to ensure the proper $V_{G S}$ voltage turning on the MOSFET, as well as to not exceed the maximum drain-to-source and gate-to-source breakdown voltages.

$$
V_{D D}<\left|V_{G S \max }\right| \text { and } V_{D D}<\left|V_{D S \max }\right| .
$$

The independent setting of voltage levels in the low VL and high VH states at the output of the EL7158 driver allows for the optimal selection of the control voltage amplitude. The EL7158 controller also has very good dynamic characteristics, the same rise and fall times, and the same delay times of switching on and off the output signal; thus, it does not introduce additional distortions of the DPWM signal at the HBS output.

At the output of the HBS, a second-order low-pass LRC filter is typically used to suppress the high-frequency components of the LBDD PWM signal and to pass the audio components to the load. The magnetic components of the output filter, which should be put as close as possible to the HBS output, increase the complexity, size, and cost of the amplifier. 


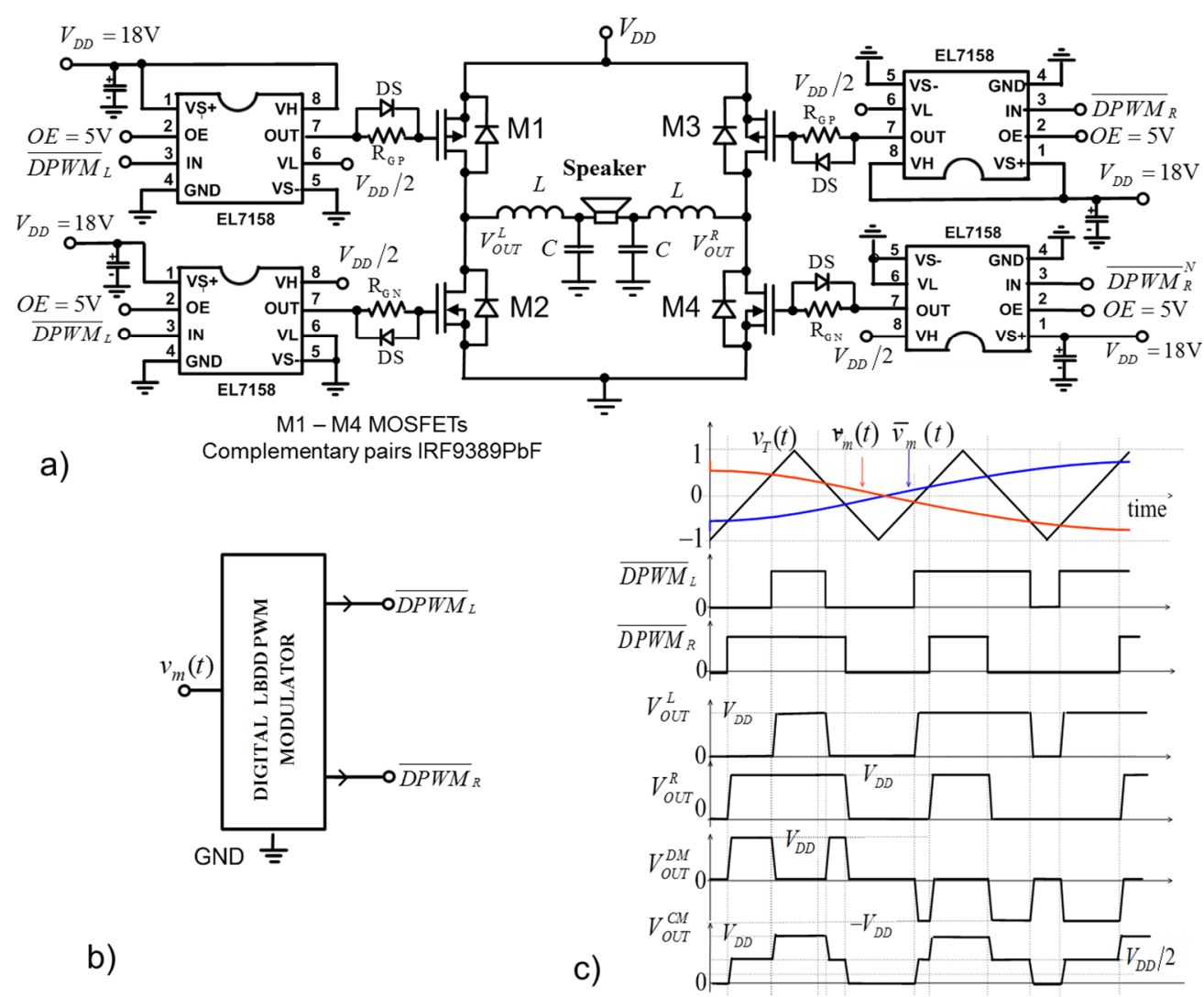

Figure 3. Digital Class-BD amplifier using LBDD PWM modulator: (a) circuit diagram; (b) block diagram of digital LBDD PWM modulator; (c) control signals and DM and CM output voltages.

\section{Filterless, Open-Loop Digital Class-BD Amplifiers with Constant Voltage at the CM Output}

Figure 4a shows an open-loop low power Class-BD amplifier with constant voltage at the CM output implemented on four ultrahigh-current EL7158 pin drivers. The EL7158 high-performance inverting drivers are controlled by the extended digital modulator based on the LBDD PWM modulation scheme (Figure $4 \mathrm{~b}$ ) and have appropriately set voltage levels on the VL and $\mathrm{VH}$ pins to obtain the necessary voltage levels at the driver outputs, depending on the status of the IN pins. The extended LBDD PWM modulator is based on the hybrid 9 bit LBDD modulator, described in Section 2, with an additional logic that generates all control signals $S_{1}, S_{2}$, and $S_{3}$. The outputs of drivers 1,2 and drivers 3,4 are connected in parallel; however, when drivers 1,3 are active with $\mathrm{OE}$ in the high state, then drivers 3, 4 with OE in the low state have the outputs placed in the three-state mode (floating). On the contrary, when drivers 3, 4 are active, the outputs of drivers 1, 2 are placed in the three-state mode.

In order to not exceed the continuous output current of the driver $(0.5 \mathrm{~A})$, with a supply voltage of $V_{D D}=18 \mathrm{~V}$, the load resistance $R_{L}$ must not be lower than $40 \Omega$. At the differential mode (DM) output and the balanced $\mathrm{CM}$ output of the amplifier, we receive near-perfect voltage waveforms, even at high switching frequency, as presented in Figure 4c.

The architecture of the second proposed amplifier, shown in Figure 5, consists of two typical, but differently powered, Class-BD amplifiers built on the IRF9389PbF complementary power MOSFET pairs. The upper H-bridge on the (M1-M4) MOSFETs is powered from the upper $V_{D D} / 2$ power supply, while the lower H-bridge on the (M5-M8) MOSFETs is powered from the lower $V_{D D} / 2$ power supply. The resultant output signals on the left $V_{\text {OUT }}^{L}$ and right $V_{O U T}^{R}$ sides of the amplifier are the result of a series summation of the output voltages of the upper and lower H-bridges, respectively, as shown in Figure 5. 


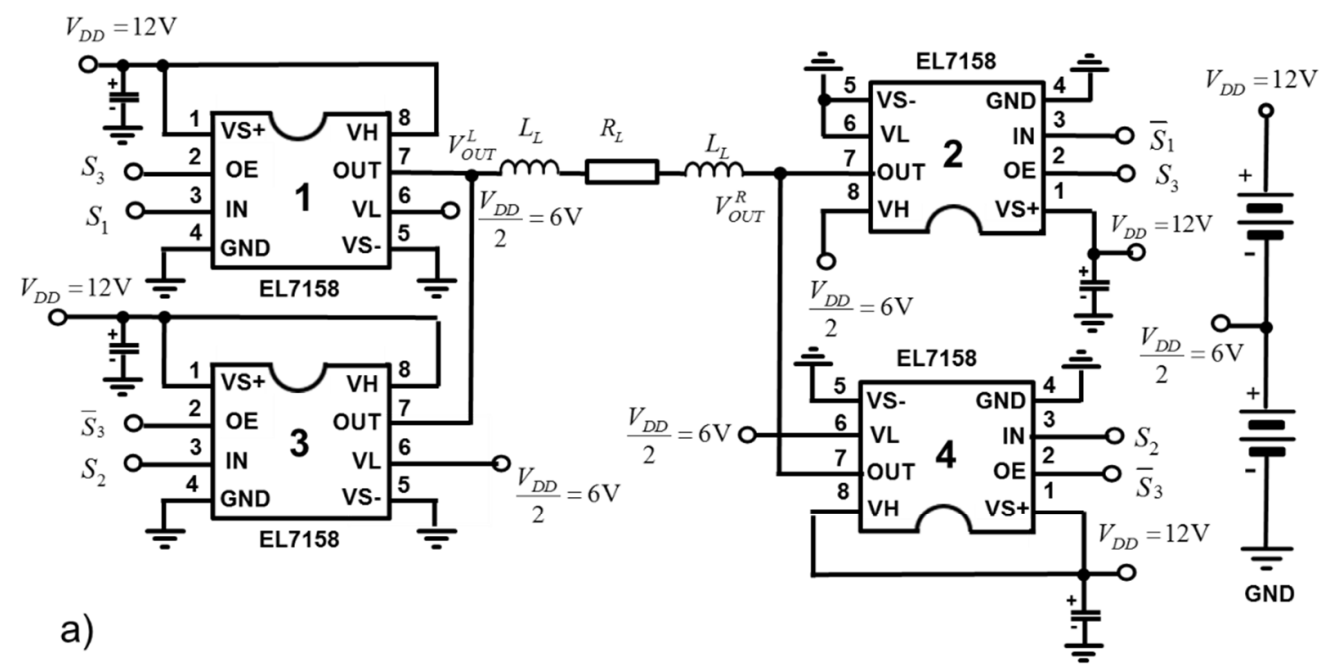

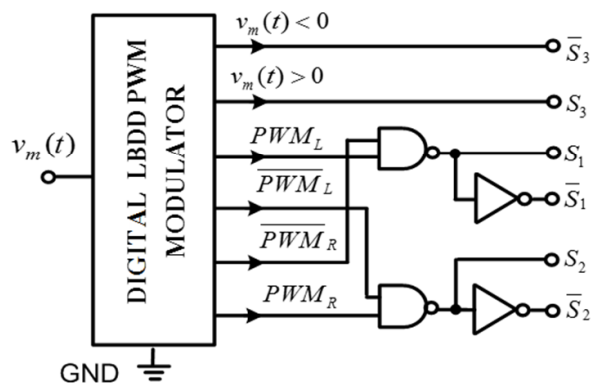

b)

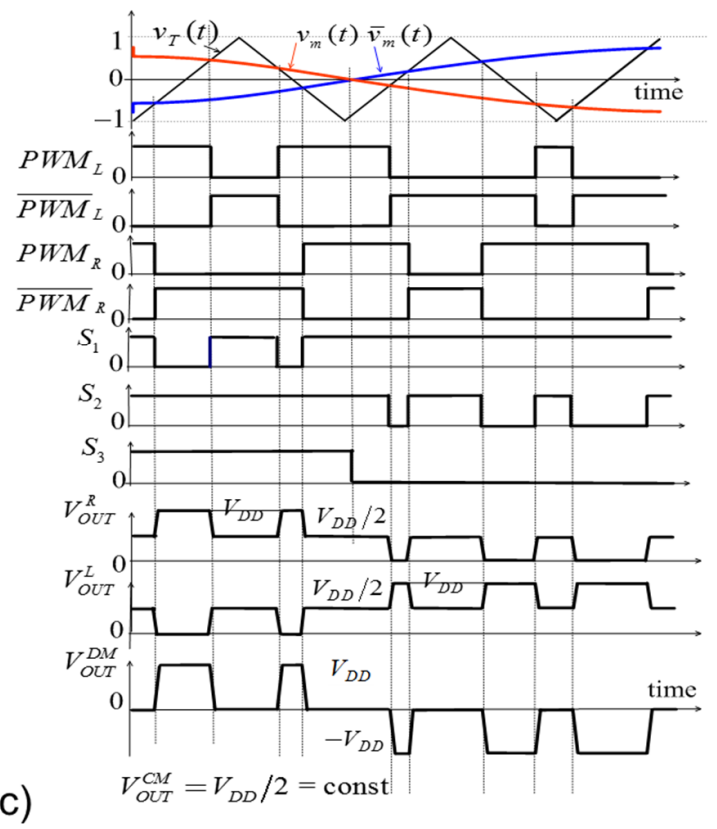

Figure 4. Filterless, digital low-power Class-BD amplifier with constant voltage at the CM output implemented on four ultrahigh-current EL7158 pin drivers: (a) circuit diagram; (b) the extended digital LBDD PWM modulator; (c) control signals and DM and CM output voltages.

Both H-bridges are driven by the EL7158 high-performance inverting drivers controlled by the extended digital modulator based on the LBDD PWM modulation, as presented in Figure $4 \mathrm{~b}$, and they have appropriately set voltage levels on the VL and VH pins to obtain the necessary voltage levels at the driver outputs, depending on the status of the IN pins. The switching frequency of the output stage MOSFETs is twice as high as the switching frequency in a conventional Class-BD amplifier (in Figure 3), since the control signals $S_{1}$ and $S_{2}$ are generated by an extended LBDD PWM modulator of this frequency. This results in higher switching power losses. In this circuit, the $S_{3}$ signals are not used, and all EL7158 drivers are active, with their OE inputs $O E=5 \mathrm{~V}$. Moreover, in this system, the set delay times before the edges of the control signals switching on the power transistors prevent the shoot-through currents and also reduce EMI. These times are independently set on the rising edges of the signals driving NMOSFETs (the $R_{G N}$ resistors) and on the falling edges of the signals driving PMOSFETs (the $R_{G P}$ resistors). The amplifier is loaded with resistance $R_{L}=4 \Omega$ or $R_{L}=8 \Omega$. At the differential mode (DM) output and the balanced CM output of the amplifier, we receive near-perfect voltage waveforms, even at high switching frequency, as presented in Figure 4c. 


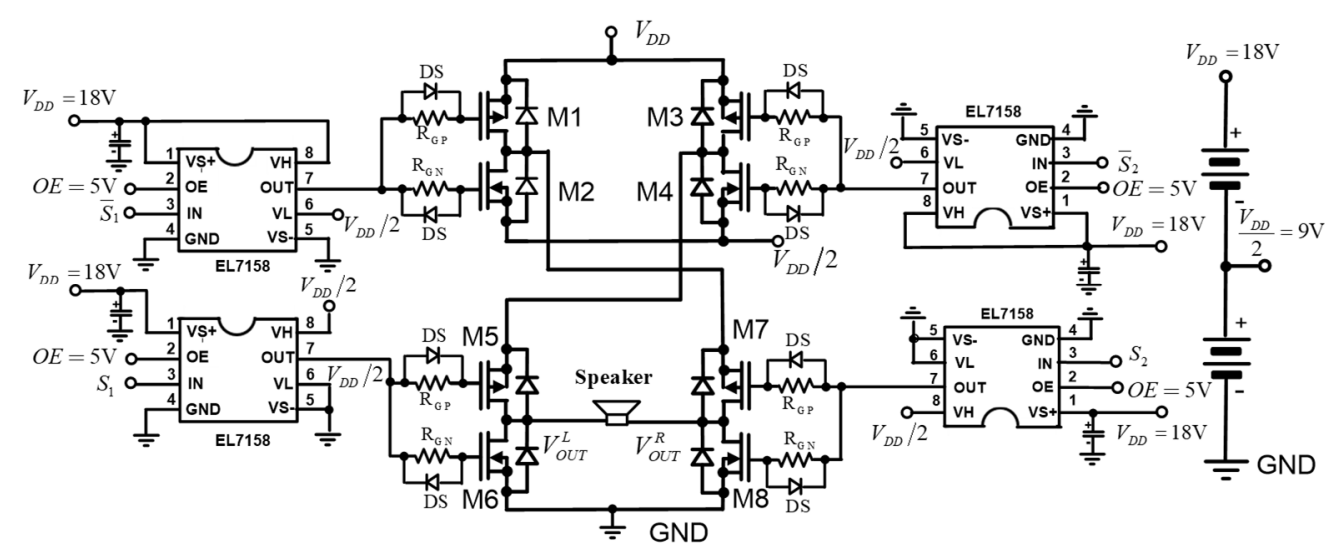

Figure 5. Circuit diagram of filterless, digital Class-BD amplifier with balanced CM output, implemented on complementary pairs of MOSFETs and four ultrahigh-current EL7158 pin drivers.

\section{Simulation and Measurement Results}

SPICE simulations and experimental results were used to compare the parameters of the proposed digital Class-BD amplifier with constant voltage at the CM output (Figure 5) with the parameters of the conventional digital Class-BD amplifier using LBDD PWM modulator (Figure 3). Both amplifiers were implemented on complementary IRF9389PbFF MOSFET pairs, driven by inverting high-performance EL7158 drivers and powered by the same voltage: $V_{D D}=18 \mathrm{~V}$. They were tested for two switching frequencies: $f_{c 1}=328 \mathrm{kHz}$ or $f_{c 2}=164 \mathrm{kHz}$, using for each of them two different load impedances: $R_{L}=4 \Omega, L=15 \mu \mathrm{H}$ or $R_{L}=8 \Omega, L=30 \mu \mathrm{H}$.

A low-power Class-BD audio amplifier with balanced CM output, implemented on four ultrahigh-current EL7158 pin drivers, was tested for $V_{D D}=12 \mathrm{~V}$ and two different load impedances: $R_{L}=40 \Omega, L=150 \mu \mathrm{H}$ or $R_{L}=80 \Omega, L=300 \mu \mathrm{H}$.

First, time domain waveforms and frequency spectrum characteristics of DPWM signals were simulated using Matlab ${ }^{\circledR}$ tools. The frequency spectrum was estimated by storing a number of pulses of the LBDD DPWM output signal, which represents more than one period of the modulating waveform. Figure 6 shows a comparison of the THD coefficients for three modulation methods, LBDD, NBDD, and UBDD, versus relative frequency $f_{r}=f_{m} / f_{c}$. This comparison shows that, even for a simple LBDD algorithm for $Q=1$ between two adjacent samples $y_{0}(n)$ and $y_{2}(n)$ of the audio signal, only one intermediate sample $y_{1}(n)$ is generated with the distance: $T_{Q}=T_{c} / 2$, and using a recursive noise shaping model with two feedback loops allows a significant improvement in the modulator's linearity.

In each $\mathrm{H}$-bridge branch of the amplifier output stage, it is necessary to insert a blanking period between either high-side turn-off and low-side turn-on or low-side turnoff and high-side turn-on when switching transistors to reduce shoot-through or a rush of current through both MOSFETs. The use of $R_{G}$ gate resistors with antiparallel Schottky diodes, as in Figures 3 and 5, allows adjustment of the MOSFET turn-on delay time ( $R_{G N}$ allows adjusting the delay time of the rising edges of the PWM signal, while $R_{G P}$ allows adjusting the delay time of the falling edges) independently. During turn-off, the antiparallel Schottky diode shunts out serial resistor $R_{G}$, providing a significant reduction in turn-off delay time. 


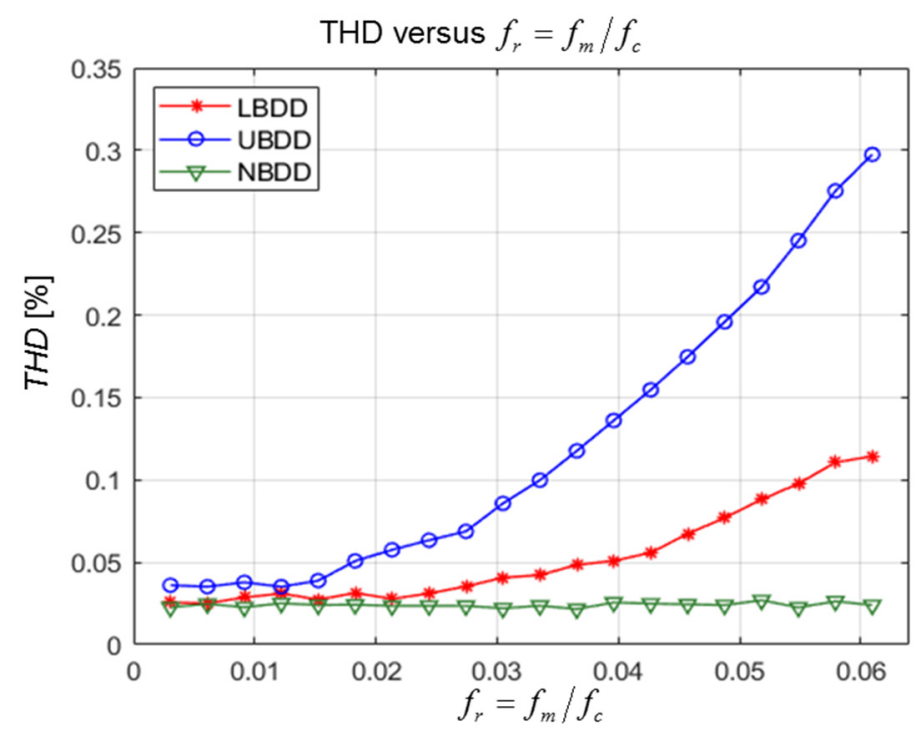

Figure 6. A comparison of the THD coefficients for three modulation methods, LBDD, NBDD, and UBDD, versus relative frequency $f_{r}=f_{m} / f_{c}$.

For optimal values of the gate resistors $R_{G N}$ and $R_{G P}$, we receive turn-on delay times $t_{d N}$ for NMOS and $t_{d P}$ for PMOS, for which a compromise is possible to obtain an acceptable level of THD and a significant limitation of the shoot-through currents. In order to determine the values of these resistances, the currents of the drains and bypass diodes of transistor pairs M1, M2 and M3, M4 in the output stage in Figure 3a were simulated in PSPICE, using the transient analysis with the "Parametric Sweep" option.

Figure 7 shows the currents of drains and diodes shunting M1 and M2 transistors in one period of the DPWM signal, when switching a constant load current, and when one of the transistors is inversely conducting. The optimal resistances $R_{G N}=110 \Omega, R_{G P}=70 \Omega$, were selected on the basis of the transient, multirun parametric analyses at the specifying sweep parameters of the gate resistors: $R_{G N}[110,140,170] \Omega$ and $R_{G P}[40,70,110] \Omega$. For the optimal resistances, the shortest turn-on delay times, $t_{d N}$ for NMOS and $t_{d P}$ for PMOS, as well the greatest limitation of the shoot-through current amplitudes, were obtained.

Figure 8 shows the SPICE-simulated time waveforms of the load current and output voltages in $\mathrm{DM}$ and $\mathrm{CM}$ modes of the Class-BD amplifier with balanced $\mathrm{CM}$ output (from Figure 5), at $R_{L}=4 \Omega, L=15 \mu \mathrm{H}$, and $f_{c}=328 \mathrm{kHz}$, as well as part of the period of the modulating signal with frequency $f_{m}=10 \mathrm{kHz}$.

The circuit has a perfectly balanced CM output with a constant voltage $V_{D D} / 2$, although very small spikes are generated at this output when switching transistors. The switching frequency of the output stage MOSFETs is twice as high $\left(2 f_{c}\right)$ as the switching frequency $f_{c}$ of the conventional Class-BD amplifier, which may result in higher switching power losses.

Figure 9 shows the output power and efficiency versus modulation index $M$, as well as THD versus the frequency of the modulating signal, of the new digital ClassBD amplifier with a balanced CM output (in Figure 5) using the extended LBDD PWM modulator compared to the conventional Class-BD amplifier (Figure 3) using an LBDD PWM modulator. The characteristics were determined for two switching frequencies, $f_{c 1}=328 \mathrm{kHz}$ or $f_{c 2}=164 \mathrm{kHz}$, using for each of them two different load impedances: $R_{L}=4 \Omega, L=15 \mu \mathrm{H}$ or $R_{L}=8 \Omega, L=30 \mu \mathrm{H}$. 


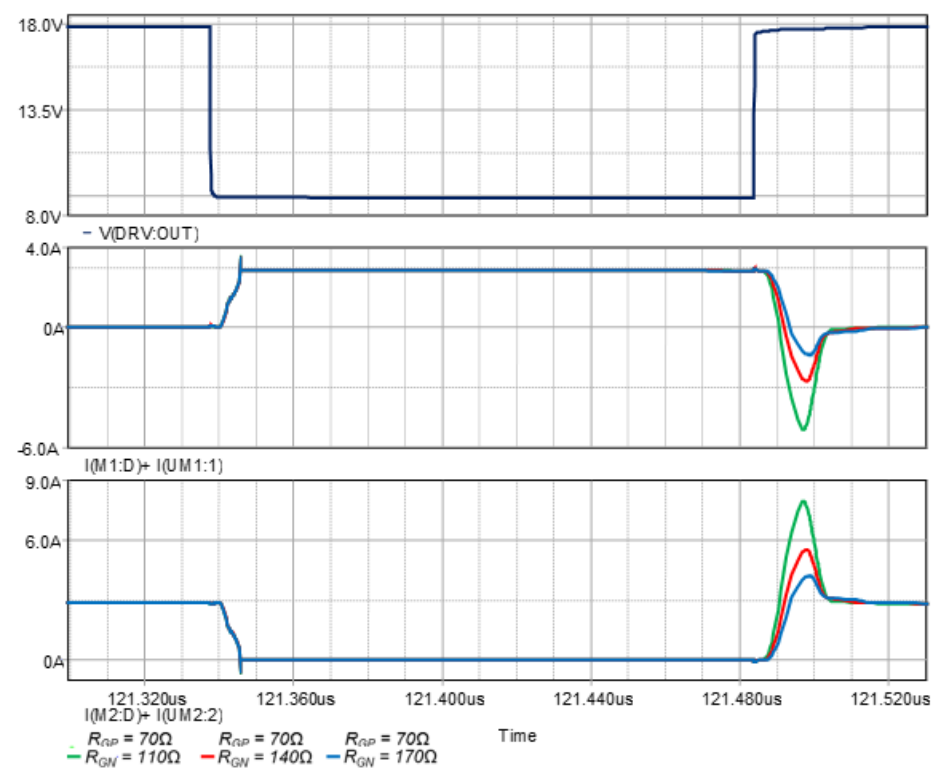

Figure 7. SPICE simulation of total currents of the drains and bypass diodes of the M1 and M2 transistors during single DPWM signal period for different turn-on delay times.
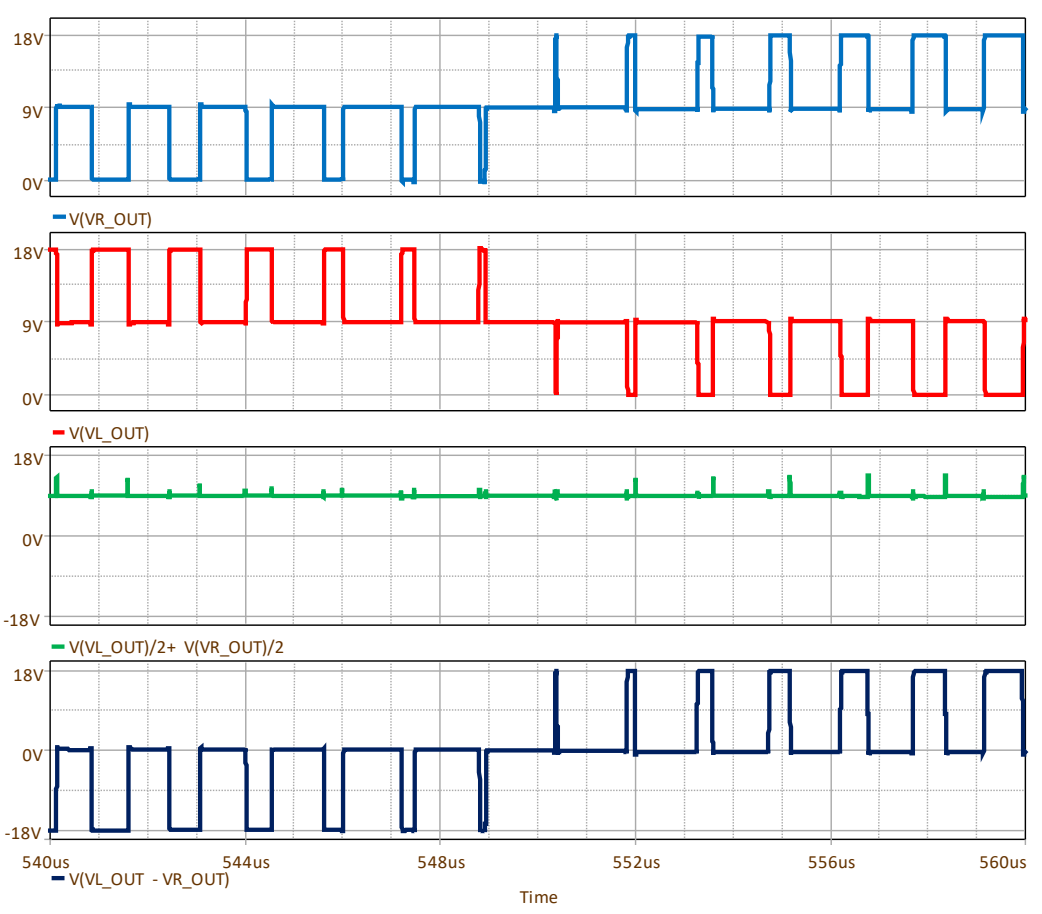

Figure 8. SPICE simulation of the load current and output voltages in DM and CM modes of the Class-BD amplifier with constant voltage at the CM output (in Figure 5).

The THD characteristics versus the $f_{m}$ frequency of the modulating signal were determined on the basis of the THD + N frequency spectra of the output voltage on the load resistance, in the band up to $30 \mathrm{kHz}$, examples of which are shown in Figure 10.

The Class-BD amplifier with constant voltage at the CM output has almost the same the output power and efficiency characteristics as the conventional one, but it exhibits a slightly higher level of nonlinear distortions, which are caused by the distorted DPWM waveform being generated in the output stage by nonideal MOSFET switches. The most significant contribution to the total THD is represented by switching timing errors added by the gate drivers, such as dead-time, $t_{o n} / t_{o f f}$, and $t_{r} / t_{f}$. The distortions are also caused by nonlinearity in the DPWM signal from the modulator to switching stage due to limited 
resolution, as well as jitter in timing. Another source of distortions is represented by unwanted characteristics in the switching devices, such as finite ON resistance (particularly when the conductive branch of the output stage contains four MOSFET switches), finite switching speed, or body diode characteristics.
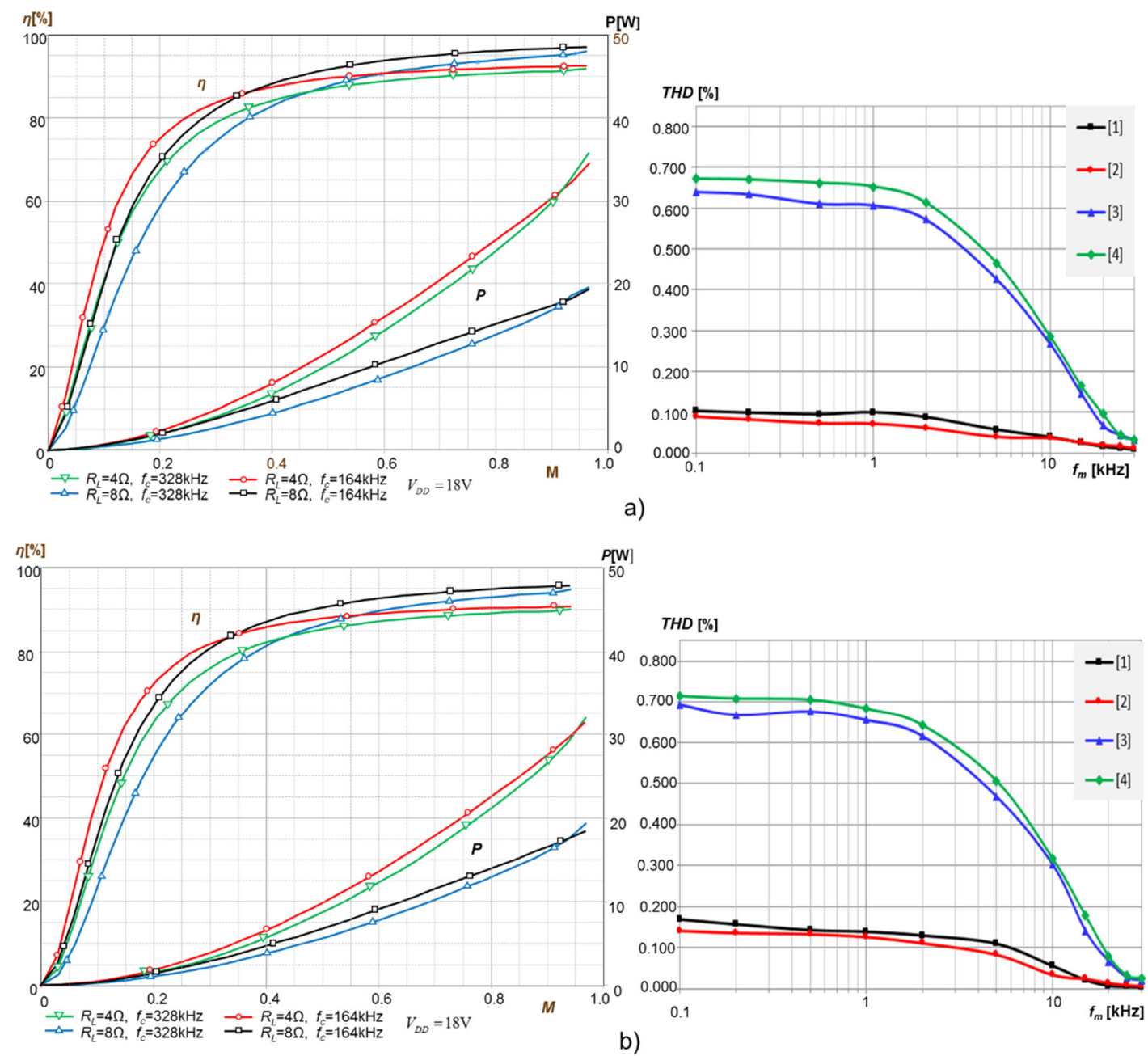

Figure 9. The output power and efficiency versus modulation index $M$ (on the left) and THD versus the $f_{m}$ frequency of the modulating signal (on the right): (a) conventional Class-BD amplifier (Figure 3); (b) Class-BD amplifier with a balanced CM output (Figure 5).

Figure 11a shows the-simulated time waveforms of the load current and output voltages in DM and CM modes of the low-power Class-BD amplifier with balanced CM output and implemented on four EL7158 drivers (Figure 4a), with $R_{L}=40 \Omega, L=150 \mu \mathrm{H}$, $f_{c}=328 \mathrm{kHz}$, and $f_{m}=10 \mathrm{kHz}$, while Figure $11 \mathrm{~b}$ shows the characteristics of the output power and efficiency versus modulation index $M$, for two switching frequencies, $f_{c 1}=328 \mathrm{kHz}$ or $f_{c 2}=164 \mathrm{kHz}$, using for each of them two different load impedances: $R_{L}=40 \Omega, L=150 \mu \mathrm{H}$ or $R_{L}=80 \Omega, L=150 \mu \mathrm{H}$. This amplifier has lower efficiency (approximately $85 \%$ ), mainly due to the conduction power losses, because the conductive branch of the output stage contains four MOSFET switches (each of them with a resistance of $0.5 \Omega$ ) connecting the output pin OUT of the driver to the input pins VH or VL, depending on the status of the IN pin. The conventional Class-BD amplifier using LBDD PWM modulator contains two MOSFET switches in the conductive branch of the output stage, which are switched at the switching frequency $f_{c}$, while the conductive branch of the output stage of Class-BD amplifier with balanced CM output, using an extended LBDD PWM modulator, contains four MOSFET switches, which are switched at the frequency of $2 f_{c}$. The practical 
implementation of this amplifier is very easy, while providing a perfectly balanced $\mathrm{CM}$ output with a constant voltage $V_{D D} / 2$.
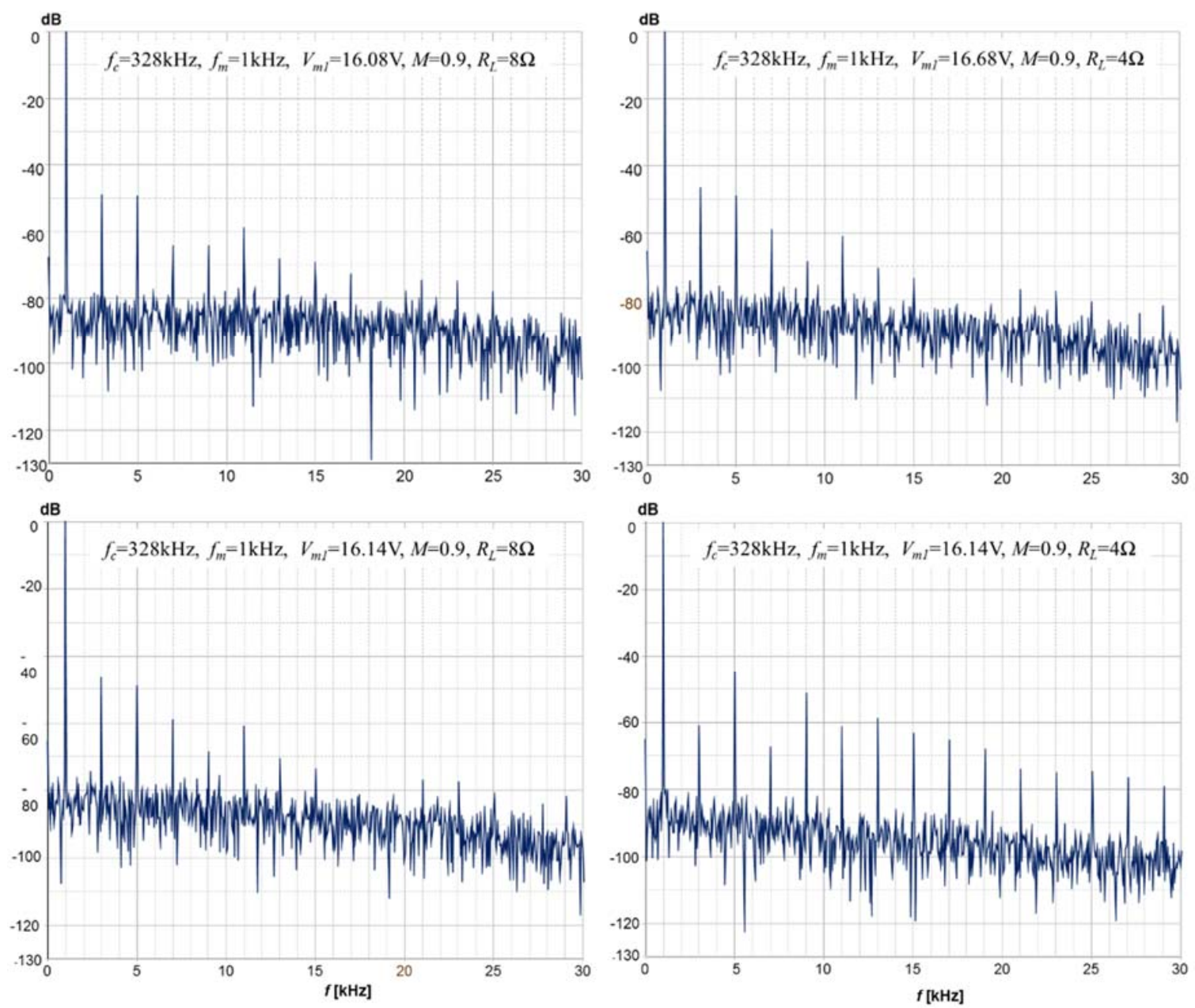

Figure 10. Simulated THD + N frequency responses of the tested amplifiers in the band up to $30 \mathrm{kHz}$.
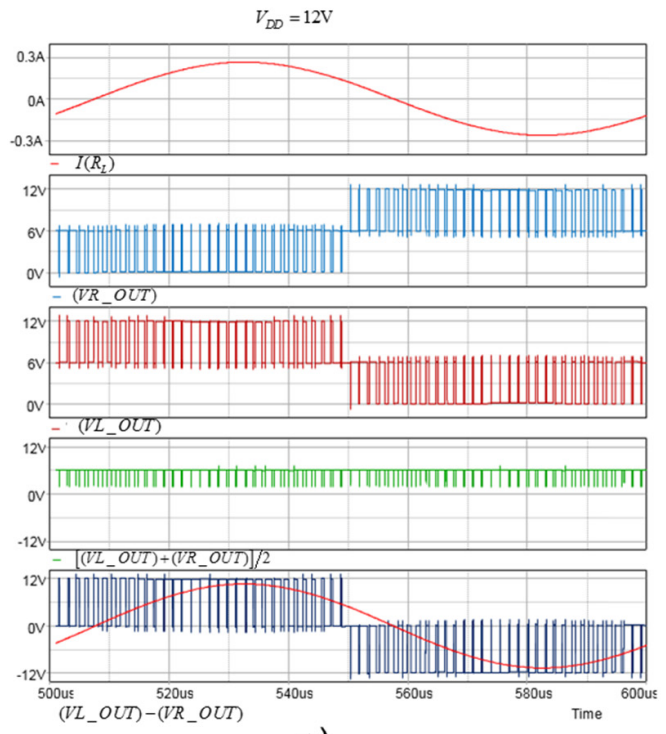

a)

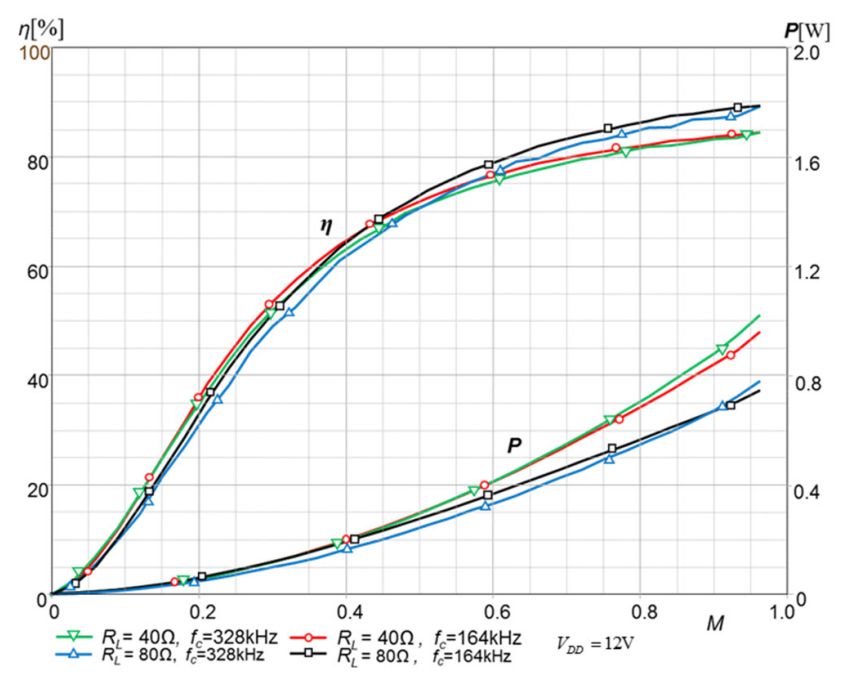

b)

Figure 11. SPICE simulations of the low-power Class-BD amplifier with constant voltage at the CM output (in Figure 4a): (a) load current and output voltages in DM and CM modes; (b) output power and efficiency versus modulation index M. 
Figure 12 shows a photograph of two PCBs containing the output stages of two tested Class-BD amplifiers: (a) conventional Class-BD amplifier (in Figure 3); (b) ClassBD amplifier implemented on complementary MOSFET pairs (in Figure 5). All gate drivers of the output stages are steered by signals generated by the digital extended LBDD PWM modulator.

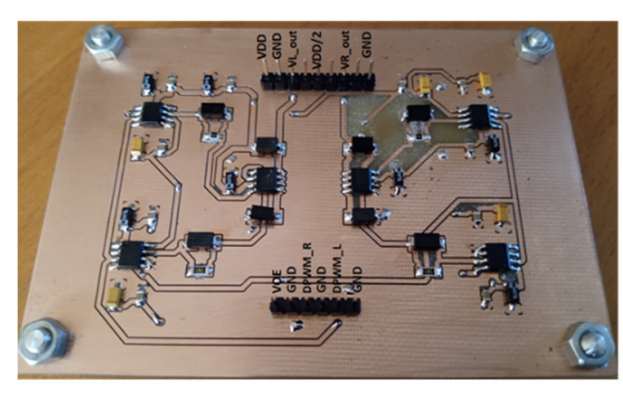

a)

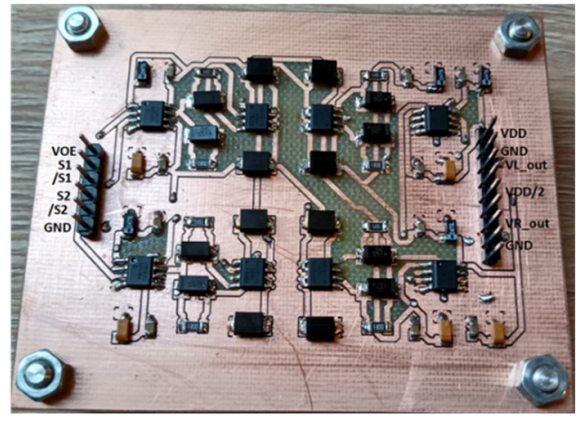

b)

Figure 12. Photographs of the two PCBs containing the output stages of (a) a conventional Class-BD amplifier (Figure 3), and (b) a Class-BD amplifier implemented on complementary MOSFET pairs (Figure 5).

Load impedances are connected to the PCBs with short cables from the outside.

The following equipment was used to test the amplifiers:

- Rigol DG1032Z function generator;

- Hp 8593 Agilent spectrum analyzer, $1 \mathrm{kHz}-2.9 \mathrm{GHz}$;

- DSO4254B advanced digital oscilloscope $4 \times 250 \mathrm{MHz}$.

Figure 13 shows the oscillograms of the control signals generated by the hybrid 9 bit LBDD PWM modulator in Figure $4 \mathrm{~b}$ with extended architecture and oscillograms of the output voltages in DM and CM modes of the Class-BD amplifier with constant voltage at the CM output (in Figure 5).

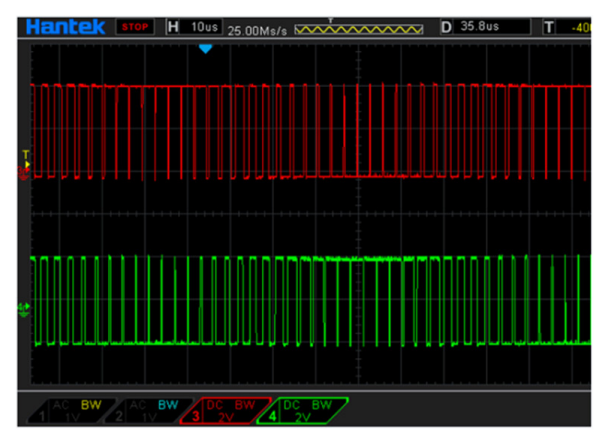

a)

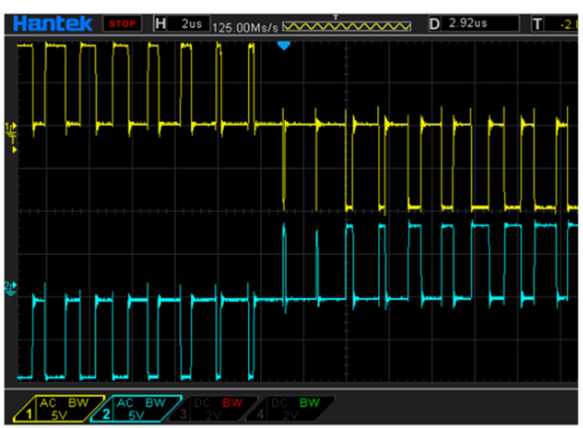

c)

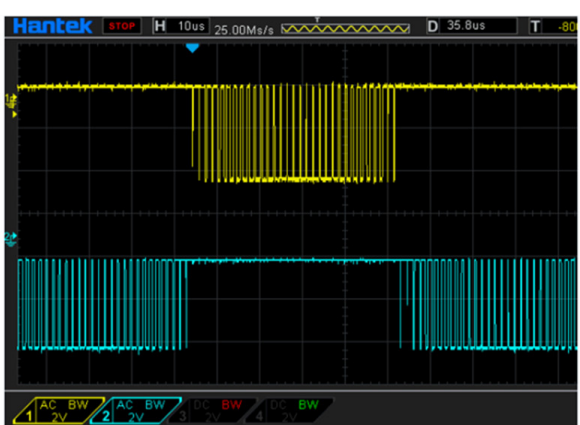

b)

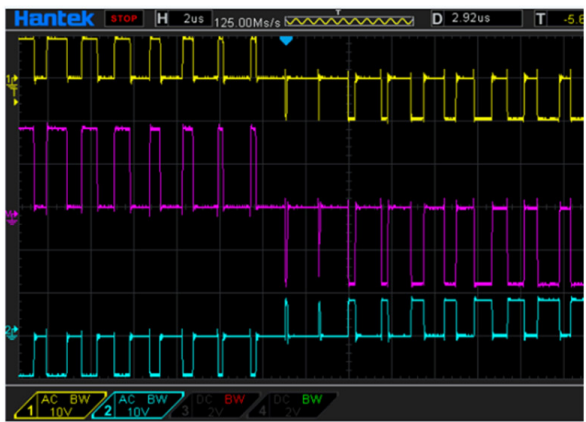

d)

Figure 13. Cont. 


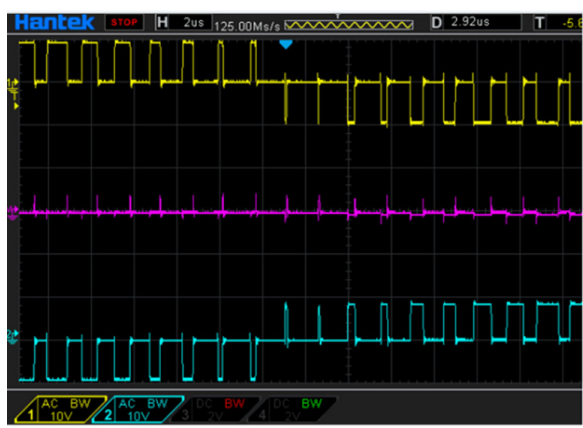

e)

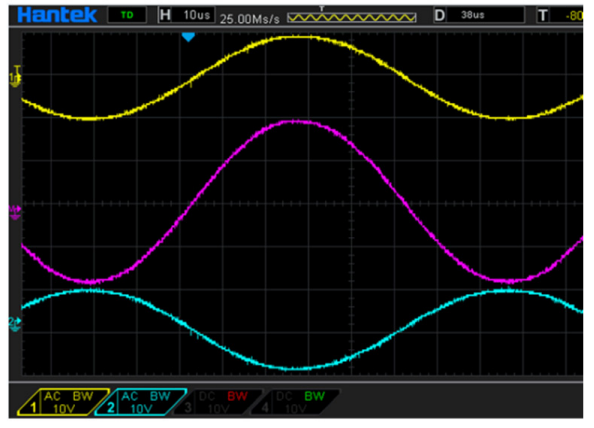

f)

Figure 13. Oscillograms of the control signals generated by the hybrid 9 bit LBDD PWM modulator in Figure $4 \mathrm{~b}$ with extended architecture with $f_{c 1}=328 \mathrm{kHz}$ and $f_{m}=30 \mathrm{kHz}$ : (a) $P W M_{L}$ and $P W M_{R}$ signals; (b) $S_{1}$ and $S_{2}$ signals; (c) output voltages in DM and CM for amplifier from Figure 5 $\left(V_{D D}=18 \mathrm{~V} ; R_{L}=8 \Omega ; L=30 \mu \mathrm{H}\right) ; V_{\text {OUT }}^{L}$ and $V_{\text {OUT }}^{R}$ voltages; (d) $V_{D M}=V_{\text {OUT }}^{L}-V_{O U T}^{R}$ voltage; (e) $V_{C M}=(1 / 2)\left(V_{\text {OUT }}^{L}+V_{\text {OUT }}^{R}\right)$ voltage; (f) voltage $V_{R_{L}}=I_{L} R_{L}$ on resistance $R_{L}$.

\section{Discussion}

We described and tested two new and original open-loop digital Class-BD audio amplifiers with constant CM output voltage. The output stage of the first, low-power ClassBD amplifier was implemented using only four ultrahigh-current EL7158 pin drivers, while the second, higher-power Class-BD amplifier was implemented using four complementary HEXFET IRF9389PbF power MOSFET pairs, formed into two cooperating and interacting typical H-bridges and driven by four ultrahigh-current EL7158 pin drivers. The drivers have appropriately set voltage levels on the VL and VH pins and are controlled by an extended LBDDPWM modulator based on a digital, hybrid 9 bit LBDD PWM modulator, described in detail in a previous study [7].

Extensive experimental and SPICE simulation studies were conducted to compare the performance of the proposed Class-BD amplifier with balanced CM output to the performance of the conventional Class-BD amplifier. As presented in Figure 9, the characteristics of the output power and the efficiency versus modulation index $\mathrm{M}$, as well as THD versus the audio signal frequency, were tested for two switching frequencies, $f_{c 1}=328 \mathrm{kHz}$ and $f_{c 2}=164 \mathrm{kHz}$, using with each switching frequency two different load resistances: $R_{L}=4 \Omega$ and $R_{L}=8 \Omega$. As we can see in Figure 9, the proposed Class-BD amplifier with a balanced CM output has a slightly better performance at the DM output than the conventional one.

For example, for $R_{L}=4 \Omega$ and $f_{c 1}=328 \mathrm{kHz}$, when adjusting the modulation index $\mathrm{M}$ from 0 to 0.96 , the load power $P_{L}$ at its differential output changes in the range from 0 to $35 \mathrm{~W}$ and is higher than the load power $P_{L}$ of a conventional Class-BD amplifier in this range from 0 up to $2.5 \mathrm{~W}$, while the efficiency $\eta$ varies from $0 \%$ to $96 \%$ and is higher in this range from $0 \%$ to $1 \%$ than the efficiency of a conventional Class-BD amplifier. For other combinations of $R_{L}$ and $f_{c}$, the load power $P_{L}$ and the efficiency $\eta$ of the Class-BD amplifier with balanced CM output are also slightly higher than for the conventional Class-BD amplifier, but with a narrower range than the example above.

In order to verify the results of the simulation tests, actual measurements of parameters were made at selected points of the simulated characteristics in Figure 9 on the model of the implemented amplifier in Figure 5 (PCB in Figure 11b). The efficiency $\eta$ was determined on the basis of the measurement of the load power $P_{L}$ in the resistance $R_{L}$ and the $P_{D C}$ power of the source powering the output stage of the amplifier, while the spectrum analyzer was used to measure the harmonic content of the output signal spectrum in the band from $30 \mathrm{~Hz}$ to $30 \mathrm{kHz}$. For example, for $R_{L}=4 \Omega$ and $f_{c 1}=328 \mathrm{kHz}$, when adjusting the modulation index $\mathrm{M}$ from 0 to 0.96 , the load power $P_{L}$ changes in the range from 0 to $30 \mathrm{~W}$ and is lower than the simulated load power $P_{L}$ in this range from 0 up to $1.5 \mathrm{~W}$, while the efficiency $\eta$ varies from $0 \%$ to $90.3 \%$ and is lower in this range from $0 \%$ to $1 \%$ than the 
simulated efficiency. For other combinations of $R_{L}$ and $f_{c}$, the measured load power $P_{L}$ and the efficiency $\eta$ are also slightly lower than simulated values but with a narrower range than the example above. The obtained measurement results are slightly worse than the simulation results, because the simulations did not take into account the resistances of the wires and paths to the power supply and the resistance of the real coil in the load.

As we can see in Figure $4 c$ and the oscillograms in Figure 13b, at the DM output and the balanced CM output of the amplifier, we receive near-perfect voltage waveforms. The upper parts of the output voltage $V_{O U T}^{L}$ and $V_{O U T}^{R}$ waveforms on the left and right branches of the output H-bridge are generated by the upper HBS on the (M1-M4) MOSFETs, while the lower parts are generated by the lower HBS on the (M5-M8) MOSFETs. Each of the three states at the DM output of the amplifier is the result of switching on only one MOSFET pair, causing low conduction power losses. Moreover, switching of each complementary pair of transistors in the upper or lower $\mathrm{H}$ bridge occurs at half the supply voltage $V_{D D} / 2$, which results in lower switching power losses than in the conventional Class-BD amplifier.

As presented in Figure 7, the THD characteristics as a function of the $f_{m}$ input signal frequency were determined on the basis of the THD $+\mathrm{N}$ frequency spectra of the output voltage on the load resistance, in the band up to $30 \mathrm{kHz}$. All tested amplifiers show some distortion at the DM outputs, with the largest for $f_{c 1}=328 \mathrm{kHz}, R_{L}=4 \Omega$ : conventional Class-BD amplifier up to $0.7 \%$, and Class-BD amplifier with constant voltage at the CM output up to $0.68 \%$. Much lower distortions, below $0.1 \%$, are obtained for both amplifiers at $f_{c 2}=164 \mathrm{kHz}$ (power MOSFET transistors, controlled by $S_{1}, S_{2}$ signals, are switched with the frequency of $2 f_{c}=328 \mathrm{kHz}$ ). These distortions are a result of the distorted LBDD PWM time waveforms generated by the output stages of these amplifiers, caused by the switching timing errors. It is well known that switching timing errors in the gate signals, due to turn-on delay times of the transistors, in particular, have the most significant contribution to nonlinearity in DM output stages.

Comparing the obtained results with the results of other studies, it can be concluded that the measured and simulated performance of the proposed amplifier is slightly better than that of similar Class-BD amplifiers with balanced CM output, described, e.g., in [10-12].

As presented in [10], a Class-BD amplifier with the power of $20 \mathrm{~W} /$ channel with almost zero CM radiation emission had the configuration of the H6-type output stage and used the PSCPWM modulation method. The H6-bridge contained two additional series switches M5 and M6 connected parallel with the load, which were used to shunt the bidirectional inductive load current when M1-M4 were off and to set both outputs at $V_{D D} / 2$ with the help of shunt resistors $R_{b}$ connected parallel with M1-M4 transistors. With switching frequency of $250 \mathrm{kHz}$, power supply of $12 \mathrm{~V}$, and $R_{L}=4 \Omega$, the amplifier could deliver $10 \mathrm{~W}$ at the efficiency $\eta$ up to $89 \%$ and THD $+\mathrm{N}$ up to $0.6 \%$, whereas, with supply of $24 \mathrm{~V}$, it could deliver $18 \mathrm{~W}$ at the efficiency $\eta$ up to $81 \%$ and THD $+\mathrm{N}$ up to $0.6 \%$. The significant reduction in efficiency was due to the additional power losses in shunt resistors $R_{b}$ connected in parallel with M1-M4 to set both outputs at $V_{D D} / 2$, which also increased the rise and fall times of the PWM output pulses, contributing to a further increase in switching power losses.

The output stage of the open-loop Class-BD audio amplifiers presented in [11,12] with balanced CM included a typical H-bridge with four MOSFETs and four additional MOSFET switches that balanced and maintained the CM output. These amplifiers employed the extended NBDD PWM or PSC PWM modulation scheme. With a switching frequency of $328 \mathrm{kHz}$, power supply of $24 \mathrm{~V}$, and $R_{L}=4 \Omega$, the amplifier could deliver $56 \mathrm{~W}$ at the efficiency $\eta$ up to $91 \%$ and THD $+\mathrm{N}$ up to $0.76 \%$, whereas, with $R_{L}=8 \Omega$, it could deliver $32 \mathrm{~W}$ at the efficiency $\eta$ up to $96 \%$ and THD $+\mathrm{N}$ up to $0.73 \%$.

The performance of the compared amplifiers is similar, but the control system for the proposed Class-BD amplifier in this paper is simpler and easier to implement. 


\section{Conclusions}

New and original architectures and implementations of two filterless, open-loop digital Class-BD audio amplifiers with constant common mode (CM) output voltage were presented.

The extended digital LBDD PWM modulator uses a linearized Class-BD double-sided modulation scheme with pre-compensation, enabling the most faithful emulation of the natural NBDD PWM and, thus, providing an attractive spectral characteristic at the DM output, similar to the NBDD PWM, while simultaneously providing a balanced CM output with constant voltage at this output. The simple filtering of the DPWM signal at the DM output by the resistive-inductive impedance of the loudspeaker and the elimination of $\mathrm{CM}$ signal filtering at the $\mathrm{CM}$ output (constant voltage at this output) are the most desirable features of a filterless Class-D amplifier without an external LC filter. The elimination of CM signals also significantly contributes to the reduction in EMI, particularly those produced on the cables connecting the loudspeaker to the HBS terminal.

Proposed amplifiers with very original topologies, due to their simple design and easy control (no galvanic isolation of control signals and no floating bias power supplies), which generate no $\mathrm{CM}$ voltage and feature excellent DM performance, similar to conventional Class-BD amplifiers, are very attractive solutions for filterless Class-BD amplifiers.

The topology of the amplifier output stage is formed by two typical $\mathrm{H}$ bridges in which MOSFET switching processes are the same as in a conventional Class-BD amplifier; therefore, it is easier and more effective to reduce the amplitudes of the shoot-through currents. Switching of each complementary pair of transistors in the upper or lower $\mathrm{H}$ bridge occurs at half the supply voltage $V_{D D} / 2$, which results in lower switching power losses than in a conventional Class-BD amplifier.

It seems that the proposed new Class-BD amplifier topology is easier to implement and more efficient than the H5, H6, and HERIC topologies that are used in transformerless full-bridge PV grid-tied inverters $[19,20]$.

Author Contributions: Conceptualization, S.K., W.M. and W.K.; methodology, W.K. and W.M.; software, W.K. and W.M.; validation, W.K. and Ł.C.; formal analysis, S.K.; investigation, W.K. and Ł.C.; resources, W.M.; data curation, W.K. and Ł.C.; writing-original draft preparation, S.K. and W.M.; writing-review and editing, W.M.; visualization, W.K. and Ł.C.; supervision, S.K.; project administration, W.K.; funding acquisition, S.K. and W.M. All authors have read and agreed to the published version of the manuscript.

Funding: This research was supported (W.M.) by the AGH University of Science and Technology, Kraków, Poland under contract 16.16.230.434. The APC was funded by the AGH University of Science and Technology, Kraków and the University of Applied Sciences in Tarnów, Poland.

Institutional Review Board Statement: Not applicable.

Informed Consent Statement: Not applicable.

Data Availability Statement: Not applicable.

Conflicts of Interest: The authors declare no conflict of interest.

\section{References}

1. Teplechuk, M.A.; Gribben, A.; Amadi, C. True Filterless Class-D Audio Amplifier. IEEE J. Solid-State Circuits 2011, 46, $2784-2793$. [CrossRef]

2. Jiang, X.; Song, J.; Cheung, D.; Wang, M.; Arunachalam, S.K. Integrated Class-D Audio Amplifier with $95 \%$ Efficiency and 105 dB SNR. IEEE J. Solid-State Circuits 2014, 49, 2387-2396. [CrossRef]

3. Zheng, H.; Zhu, Z.; Ma, R. A 0.02\% THD and $80 \mathrm{~dB}$ PSRR filterless class D amplifier with direct lithium battery hookup in mobile application. J. Semicond. 2017, 38, 56-63. [CrossRef]

4. He, H.; Kang, Y.; Yu, J. A novel low-power high-efficiency 3-state filterless bang-bang class D amplifier. In Proceedings of the IEEE International Conference on Electronics, Circuits, and Systems (ICECS), Cairo, Egypt, 6-9 December 2015; pp. 93-96.

5. Chien, S.-H.; Chen, Y.-W.; Kuo, T.-H. A low quiescent current, low THD+N class-D audio amplifier with area-efficient PWMresidual-aliasing reduction. IEEE J. Solid-State Circuits 2018, 53, 3377-3385. [CrossRef] 
6. Johansen, M.; Nielsen, K. A Review and Comparison of Digital PWM Methods for Digital Pulse Modulation Systems. In Proceedings of the 107th AES Convention, New York, NY, USA, 24-27 September 1999.

7. Kołodziejski, W.; Kuta, S. Linearized 9-Bit Hybrid LBDD PWM Modulator for Digital Class-BD Amplifier. Int. J. Electron. Telecommun. 2021, 67, 49-57.

8. Chen, X.; Qu, H.; Yu, Z.; Zhang, C.; Zhang, E. A filterless digital audio class-D amplifier based on grow-left double-edge pulse width modulation. In Proceedings of the 2nd International Conference on Integrated Circuits and Microsystems, Nanjing, China, 8-11 November 2017; pp. 251-255.

9. Li, L.; Li, H.-j.; Sun, Y.-J. Improved Error Correction Methods for Filterless Digital Class D Audio Power Amplifier Based on FCLNF. Math. Probl. Eng. 2020, 2020, 5914062. [CrossRef]

10. Siniscalchi, P.P.; Hester, R.K. A 20W/channel class-D amplifier with near-zero common-mode radiated emissions. IEEE J. Solid-State Circuits 2009, 44, 33264-33271. [CrossRef]

11. Kołodziejski, W.; Kuta, S.; Jasielski, J. Open-Loop Class-BD Audio Amplifiers with Balanced Common Mode Output. Electronics 2021, 10, 1381. [CrossRef]

12. Jasielski, J.; Kołodziejski, W.; Kuta, S. Cancellation of common-mode output signal in Class-BD audio amplifiers. In Proceedings of the International Conference on Signals and Electronic Systems (ICSES), Krakow, Poland, 5-7 September 2016; pp. 167-172.

13. AN-1497 Filterless Class D Amplifiers, Texas Instruments, Application Report, SNAA034A-May 2006-Revised May 2013. Available online: https://www.ti.com/lit/an/snaa034a/snaa034a.pdf (accessed on 15 August 2021).

14. Lin, C.W.; Hsieh, B.-S.; Lin, Y.C. Enhanced Design of Filterless Class-D Audio Amplifier. In Proceedings of the 2009 Design, Automation \& Test in Europe Conference \& Exhibition, Nice, France, 20-24 April 2009.

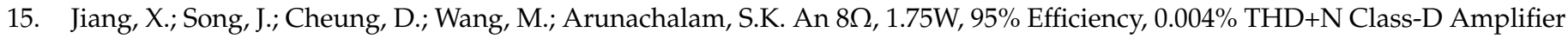
with a Feed-Forward ADC and Feedback Filters. In Proceedings of the IEEE Asian Conference on Solid-State Circuits (A-SSCC), Singapore, 11-13 November 2013; pp. 369-373.

16. Jin, C.; Tan, M.T.; See, K.Y. Filterless Class-D Amplifier with Pseudorandomized Carrier Frequency Modulation for EMI Reduction. IEEE Trans. Electromagn. Compat. 2013, 55, 74-80. [CrossRef]

17. 3-Bit Programmable FAST TTL Logic Delay Line. Available online: https://www.cornucopiaplastics.com/documents/PDL_ TTL_7_XX.pdf (accessed on 15 August 2021).

18. STM32F4 Reference Manual. Available online: https://www.st.com/ (accessed on 15 August 2021).

19. Zhang, L.; Sun, K.; Xing, Y.; Xing, M. H6 Transformer Less Full-Bridge PV Grid-Tied Inverters. IEEE Trans. Power Electron. 2014, 29, 1229-1238. [CrossRef]

20. Yu, W.; Lai, J.; Qian, H.; Hutchens, C. High-Efficiency MOSFET Inverter with H6-Type Configuration for Photovoltaic Nonisolated AC-Module Applications. IEEE Trans. Power Electron. 2011, 26, 1253-1260. [CrossRef] 